\title{
Estudio de la modificación de la microestructura de polímeros porosos por metalización mediante STM y AFM
}

\begin{abstract}
G. ARRANZ, L. PALACIO, P. PRÁDANOS, A. HERNÁNDEZ
Grupo de Superficies y Materiales Porosos (SMAP), Dpto. Termodinámica y Física Aplicada, Universidad de Valladolid, 47071 Valladolid, España.

En este trabajo se ha comparado los resultados de caracterización de membranas de microfiltración por diversas técnicas microscópicas: Microscopía Electrónica de Barrido (SEM), Microscopía de Efecto Túnel (STM) y Microscopía de Fuerza Atómica (AFM). Se ha determinado la distribución superficial de tamaños de poro y la rugosidad superficial de cada una de las membranas estudiadas. Los resultados muestran que el efecto de la capa de recubrimiento de oro, necesaria en SEM y STM, no afecta significativamente a la distribución de tamaños de poro en este tipo de membranas. Sin embargo el efecto de la capa de recubrimiento disminuye de forma general la rugosidad de la superficie.
\end{abstract}

Palabras clave: Membranas de Microfiltración, Microscopía de Fuerza Atómica, Microscopía de Efecto Túnel, Microscopía Electrónica de Barrido, Distribución de Tamaños de Poro, Rugosidades.

\section{Study of the microstructural modification by metallization of porous polymers by stm and afm}

The results of the characterization of microfiltration membranes by different microscopic techniques: Scanning Electron Microscopy (SEM), Scanning Tunnelling Microscopy (STM) and Atomic Force Microscopy (AFM), have been compared. Pore size distribution on the surface and the surface roughness have been determine for each studied membrane. The results show that the effect of the gold layer, needed in SEM and STM, does not affect significatively the pore size distribution for these membranes. However, the effect of this layer decreases the surface roughnes.

Keywords Microfiltration membranes, Atomic Force Microscopy, Scanning Tunnelling Microscopy, Scanning Electron Microscopy, Pore Size Distribution, Roughness.

\section{INTRODUCCIÓN}

Existe una gran cantidad de técnicas que habitualmente se utilizan en la caracterización de la estructura porosa de materiales poliméricos $\mathbf{( 1 , 2 ) . ~ U n o ~ d e ~ l o s ~ m e ́ t o d o s ~ m a ́ s ~ h a b i t u a l e s ~ e n ~ e s t e ~ t i p o ~ d e ~ e s t u d i o s , ~ s o - ~}$ bre todo con el fin de obtener la distribución superficial de tamaños de poro en membranas, es la microscopía. Entre estas técnicas microscópicas, se puede hablar de Microscopía Electrónica de Barrido (SEM), de Microscopía de Efecto Túnel, (STM) y de Microscopía de Fuerza Atómica (AFM), (3-5). En los dos primeros casos, debido a la baja conductividad de electrones secundarios producidos por estos materiales poliméricos, se hace necesario la deposición de una capa más o menos gruesa de un material conductor. Evidentemente, esto puede producir una modificación no deseable de la estructura. La importancia de esta modificación debe ser analizada en función de la magnitud de los elementos topográficos que se desean analizar. En el caso de la caracterización superficial de membranas poliméricas normalmente se estudian aspectos como la distribución de tamaños de poro, relacionado con la retención de la membrana; y la rugosidad de la superficie, relacionada con la intensidad y rapidez de ensuciamiento superficial en los procesos de separación (6).

En el caso de la obtención de la distribución de tamaños de poro, la utilidad de las técnicas (SEM) y (STM), dependerá de la reducción u oclusión de los poros por la capa conductora de recubrimiento. Normalmente en los trabajos publicados sobre caracterización de membranas de microfiltración (tamaños medios de poro entre 0,05 $\mu \mathrm{m}$ y varias micras), se emplean capas de recubrimiento entre 10 y 20 $\mathrm{nm}$, y no se tiene en cuenta esta reducción del tamaño de poro (7).

Por otra parte, las técnicas de Microscopía de Sonda de Barrido: STM y AFM, pueden tener el problema de la interacción entre la punta y la muestra. $\mathrm{Al}$ poseer esta punta una geometría y un tamaño determinados, su interacción con una muestra puede proporcionar resultados de tamaños de poro menores que los reales. Evidentemente, la magnitud de este efecto dependerá de los tamaños relativos de los poros y la punta y de la agudeza de ésta. En el caso de membranas de microfiltración la comparación de resultados de AFM con otras técnicas de caracterización demuestran que este efecto es totalmente despreciable (8).

En este trabajo se pretende comprobar el efecto de las capas conductoras de recubrimiento en membranas de microfiltración, comparando resultados de SEM y STM con resultados de AFM. Estos últimos serán tomados como resultados patrón tanto para los estudios de distribución de tamaños de poro, como para el análisis de la rugosidad superficial.

\section{MATERIALES Y MÉTODOS EXPERIMENTALES}

\subsection{Membranas}

Hemos seleccionado para su caracterización membranas cuya aplicación típica, dado su tamaño de poro, son los procesos de microfiltración. Se trata de membranas sintéticas que podemos agrupar en dos bloques:

- Membranas poliméricas de microfiltración de geometría irregular, todas ellas con tamaños de poro nominales de 0,2 y 0,45 $\mu \mathrm{m}$. Fabricadas por Gelman Sciences: HT Tuffryn de polisulfona hidrofílica (TF02 y TF045), GH Polypro de polipropileno hidrofílico (GH02 y GH045), Supor de polietersulfona (SU02 y SU045). Fabricadas por Sartorius: Sartolon de nylon 66 (ST02 y 
ST045). Fabricadas por Spectrum: Micropor de nylon con carga positiva (SP02 y SP045)

- Membranas orgánicas de policarbonato de poros cilíndricos paralelos, baja porosidad y distribución estrecha (Cyclopore ${ }^{\mathrm{TM}}$ ), producidas por bombardeo de trazas nucleares y posterior revelado: C01 $(0,1 \mu \mathrm{m})$, C02 $(0,2 \mu \mathrm{m}), \mathrm{C} 04(0,4 \mu \mathrm{m})$, C06 $(0,6 \mu \mathrm{m})$ C08 $(0,8 \mu \mathrm{m})$ y $\mathrm{C} 10(1,0 \mu \mathrm{m})$

\subsection{Preparación de las muestras}

Para las tres técnicas, las muestras se adhieren a un disco de acero de $10 \mathrm{~mm}$ de diámetro. Un lote de ambas caras de las membranas mencionadas se utilizan para las medidas de AFM como referencia. Otro lote similar es recubierto con una capa de oro de $10 \mathrm{~nm}$ de espesor. Además, algunas de las muestras han sido recubiertas con una capa de oro de $20 \mathrm{~nm}$ para acentuar el efecto de la capa de recubrimiento. Con el fin de asegurar la conductividad eléctrica entre la superficie de la membrana y la base de acero, se han trazado una serie de puentes metálicos entre la muestra y el soporte usando una laca conductora enriquecida con plata de alta pureza.

\subsection{Dispositivos experimentales y método de trabajo}

Las membranas han sido recubiertas con oro mediante evaporación, utilizando un metalizador Balzers (SCD 004). El proceso requiere introducir la muestra, pegada a su soporte metálico, en una cámara de vacío cuya atmósfera de argón se mantiene a una presión de $510^{-2}$ atm. El recubrimiento de $10 \mathrm{~nm}$ de espesor requirió una intensidad de corriente de 20-30 mA durante un tiempo de 85 segundos. Para el recubrimiento de $20 \mathrm{~nm}$ se duplicó el tiempo, manteniendo la misma intensidad de corriente.

Las membranas recubiertas con oro se han observado y fotografiado con un microscopio electrónico de barrido (SEM) JEOL (JSM-T300). Se ha utilizado un pedestal de alta resolución y se han visualizado las muestras con una corriente de $20 \mathrm{kV}$, consiguiendo aumentos en un rango de X5000 hasta X20000.

El microscopio de efecto túnel y fuerza atómica (STM-AFM) empleado fue un Nanoscope-IIIA de Digital Instruments. En Microscopía de Efecto Túnel se han usado puntas de una aleación de platino-iridio, mientras que en Microscopía de Fuerza Atómica se emplearon puntas y brazos de silicio con una frecuencia de aproximadamente $350 \mathrm{kHz}$.

En la adquisición de imágenes STM-AFM se han realizado medidas de ambas caras de la membrana con tamaños de barrido desde 15 $\mu \mathrm{m}$ hasta $250 \mathrm{~nm}$. Las medidas de AFM se han realizado en modo de contacto intermitente, "tapping", tanto para las membranas con recubrimiento de oro como sin él. En STM (para las membranas recubiertas de oro), las medidas se han realizado en modo de corriente constante, siendo desaconsejable el método de distancia constante debido a las grandes irregularidades que aparecen en la superficie.

Tanto las imágenes SEM como las de AFM y STM, han sido analizadas con un programa de tratamiento de imágenes, (Jandel $\circledR^{\circledR}$ ScanPro, versión 3.00.0030). Esto nos ha permitido obtener parámetros como distribución de área superficial de poros, diámetros de poro, etc.

\section{RESULTADOS Y DISCUSIÓN}

Como se ha dicho, en este trabajo se han tomado imágenes AFM en modo contacto intermitente (Tapping) de las membranas mencionadas $\sin$ recubrimiento por ambas caras, para ser usadas como referencia. Por otro lado, de las membranas recubiertas se han tomado las mismas imágenes por las técnicas de AFM-Tapping, STM y SEM. Se analizaron muestras con recubrimiento de espesor de $10 \mathrm{~nm}$ y de $20 \mathrm{~nm}$, sin apreciarse diferencias. Los resultados aquí presentados corresponden a las membranas con capa de oro de $10 \mathrm{~nm}$. Respecto a la distribución de tamaños de poro obtenidas con las distintas técnicas, podemos decir que, las diferencias encontradas no son significativas. Para las membranas de poro mayor estas diferencias se deben más a la variabilidad de la muestra o de la zona de medida que al efecto de la capa de recubrimiento o de la técnica empleada, como se aprecia en la figura 1. En ésta se muestran las imágenes comparativas de la membrana C01 por la técnica de STM y SEM de las membranas recubiertas; y por la técnica de AFM-Tapping, tanto para la membrana recubierta como sin recubrir. Las imágenes corresponden a una de las membranas con tamaño de poro más pequeño de las estudiadas, $0,1 \mu \mathrm{m}$, y por tanto, la que más podría acusar las diferencias en la distribución de tamaño de poro.

En la figura 2, se muestra la membrana SU045, $(0,45 \mu \mathrm{m}$ de tamaño medio nominal), con AFM-Tapping, de la membrana sin recubrir y recubierta de oro, SEM y STM. Se puede apreciar que igual que en el caso anterior, hay diferencias en la superficie. Parece claro que, en parte, las diferencias si son atribuibles a la técnica empleada.

En la figura 3, se representan las distribuciones de tamaños de poro, obtenidas para la membrana C01, mediante análisis de imagen con STM y AFM-Tapping (recubierta y sin recubrir). Tomando como patrón la curva obtenida mediante AFM en modo tapping, sin recubrir de oro, se observa que cuando la membrana está recubierta con oro se produce un desplazamiento, no muy importante, del máximo hacia tamaños más pequeños de poro. La microscopía STM provoca un incremento en el tamaño de poro con un aumento en la altura del pico de la distribución. No obstante estas variaciones en el tamaño de poro medio menor que el propio espesor de la capa de recubrimiento.

Tanto la microscopía de efecto túnel (STM) como la microscopía de fuerza atómica (AFM), permiten determinar de forma cuantitativa la rugosidad de un área superficial de la muestra. Las medidas muestran que las microscopías AFM y STM conducen a resultados muy similares entre sí. La rugosidad encontrada, por ejemplo, en la membrana C01 recubierta, es $R_{a}=10,0 \mathrm{~nm}$ para $A F M, y R_{a}=10,7 \mathrm{~nm}$ para $S T M$. El recubrimiento con oro hace que la membrana sea más plana y suave; ésta es una conclusión que se puede obtener si tenemos en cuenta que el resultado obtenido para la rugosidad sin recubrimiento es: $R_{a}=12,7 \mathrm{~nm}$. Es evidente que las dos técnicas dan resultados muy similares y que el recubrimiento con oro suaviza la superficie de la membrana $(\mathbf{9}, \mathbf{1 0})$.

\section{CONCLUSIONES}

Como consecuencia de este trabajo, se puede establecer una serie de conclusiones que se resumen en los siguientes enunciados:

- Se ha observado que el recubrimiento produce una disminución en la rugosidad; la superficie de la membrana se hace más suave y plana.

- El recubrimiento con oro produce una disminución en el tamaño de poro pero no es tan importante como para pensar que la capa de oro pudiera cerrar significativamente los poros. En cualquier caso no se han obtenido desviaciones mayores de un 5\% en los tamaños de los poros.

- Las variaciones en el tamaño de poro parecen más influidas por la técnica microscópica utilizada que por la capa de oro depositada sobre la membrana.

Como conclusión general podemos decir que: 

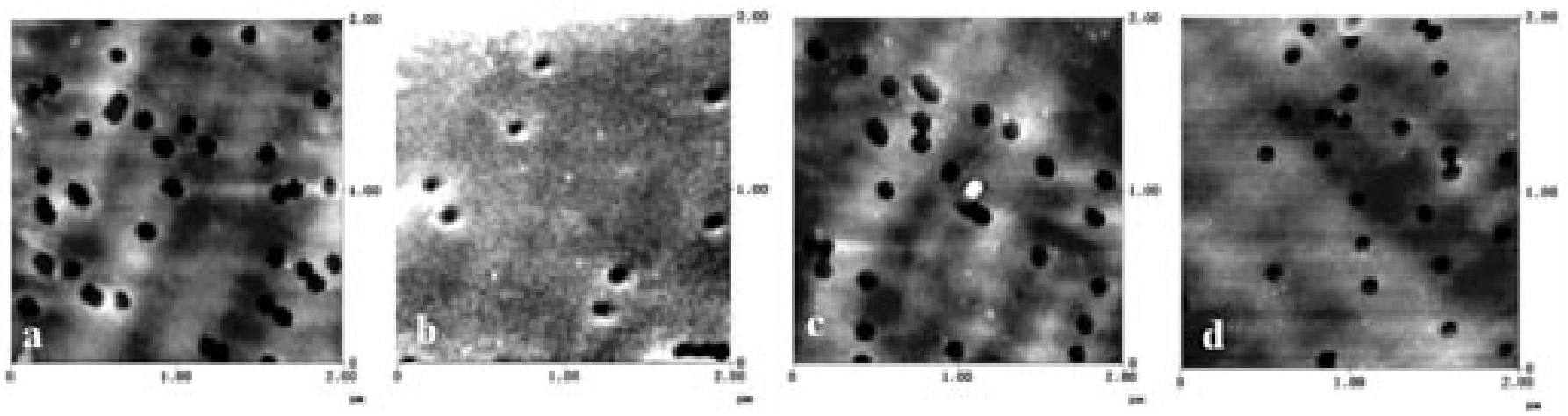

Fig. 1.- Imágenes de microscopía de la membrana C01 a) STM b) SEM c) AFM-tapping con recubrimiento de oro d) AFM-tapping sin recubrir
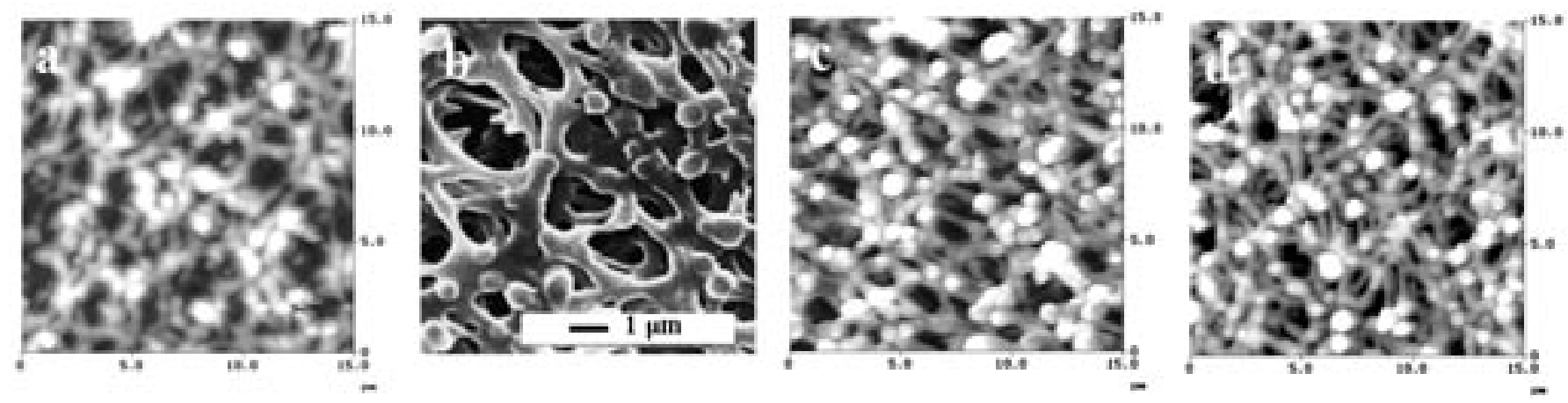

Fig. 2.- Imágenes de microscopía de la membrana SU045 a) STM b) SEM c) AFM-tapping con recubrimiento de oro d) AFM-tapping sin recubrir

- En membranas de microfiltración todas las técnicas empleadas conducen a resultados similares en la distribución de tamaño de poro.

- El efecto del recubrimiento influye en factores como la rugosidad superficial, que resulta suavizada.

- Si el tamaño de poro de una membrana es próximo al espesor de la capa de oro utilizada en el recubrimiento pueden producirse variaciones importantes en la distribución de los poros. Por ello, las técnicas de SEM y STM, cuando necesitan recubrimiento, no serán útiles en la caracterización de membranas de ultra y nanofiltración.

\section{5.- AGRADECIMIENTOS}

Los autores agradecen la financiación a los siguientes proyectos de investigación: MAT1999-0989 del Plan Nacional de Investigación y Desarrollo y VA62-00B de la Junta de Castilla y León.

\section{6.- REFERENCIAS}

1.-A. Hernández, P. Prádanos, J.I. Calvo, and L. Palacio, "Metal Oxide Membranes", pp. 355-409 en "Oxide Surfaces", (Ed. J.W. Wingrave), Marcel Dekker, Nueva York, EE.UU., 2001.

2.-J.I. Calvo, L. Palacio, P. Prádanos, A. Martín, F. Martínez, A. Hernández, "Estudio estructural de membranas Cyclopore de microfiltración", Bol. Soc. Esp. Ceram. V., 39 [4] 520-524 (2000).

3.-N.J. Dinardo, "Nanoscale characterization of surfaces and interfaces". VCH, New York 1994.

4.-H.J. Güntherodt, R. Wiesendanger, “Scanning Tunneling Microscopy I. General Principles and Applications to clean and absorbate-covered surfaces", Springer-Verlag, New York, 1994.

5.-H.J. Güntherodt, R. Wiesendanger, "Scanning Tunneling Microscopy II. Further Applications and related scanning techniques", Springer-Verlag, New York, 1995.

6.-A. Hernández, P. Prádanos, J.I. Calvo, L. Palacio, “Membranas Cerámicas y

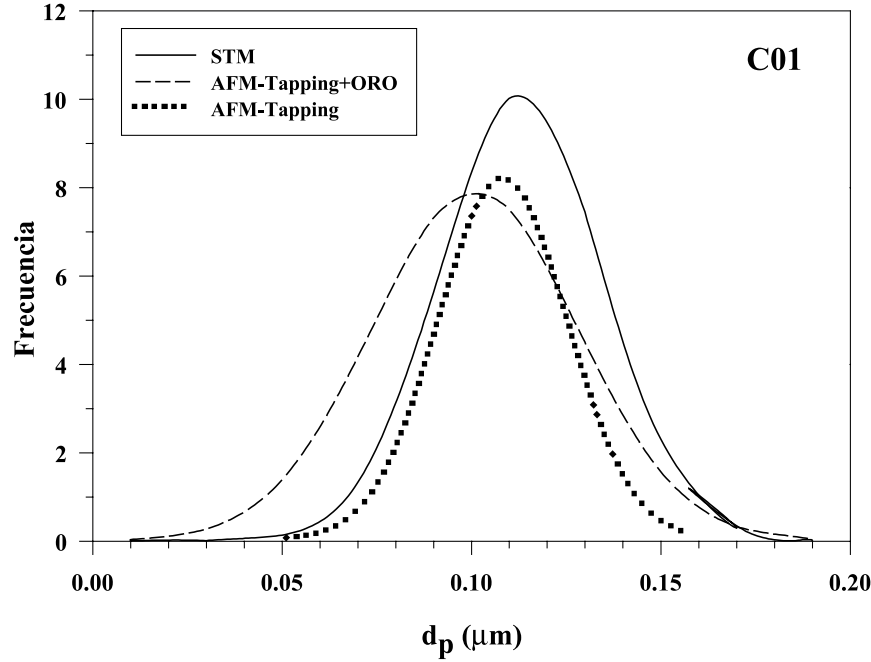

Fig. 3.- Distribución de tamaños de poro de la membrana C01 obtenida por análisis de imagen de las microfotografías de STM, AFMTapping con recubrimiento de oro y AFM-Tapping sin recubrir.

su utilidad en procesos de separación", Bol. Soc. Esp. Ceram. V., 38 [3] 185193 (1999).

7.-A.Hernández, J.I. Calvo, P. Prádanos, F. Tejerina, "Pore size distributions of track-etched membranes: comparison of surface and bulk porosities". Colloids and Surfaces A., 138 (1998) 391.

8.-L. Palacio, “Caracterización estructural y superficial de membranas microporosas". Tesis Doctoral. Universidad de Valladolid, 1998.

9.-G. Arranz, L. Palacio, J.I. Calvo, P. Prádanos, A. Hernández, “Structural characterization of microfiltration membranes by scanning tunneling microscopy". Proceedings del III Congreso Ibero-Americano en Ciencia y Tecnología de Membranas (Eds. A.M. Mendes, M.N. Pinho, F.D. Magalhaes), Aveiro, 2001.

10.-G. Arranz, L. Palacio, P. Prádanos, A. Hernández, “Topographic changes in SEM preparation of microfiltration membranes analysed by SPM". Advanced Membrane Technology Conference, Barga (Italia), 2001 